Enzyme preparation: Human Fhit protein was overexpressed in Escherichia coli as described. ${ }^{[3 a]}$ Purification from the crude bacterial extract comprised ammonium sulfate fractionation, ion-exchange chromatography on DEAE-Sephacel, gel filtration on Sephadex G-100, and immobilizedmetal-affinity chromatography on TALON (Clonetech) resin from which adsorbed protein was eluted with imidazole $(50 \mathrm{~mm})$. Homogeneous dinucleoside triphosphate hydrolase from yellow lupine seeds was obtained as described previously. ${ }^{[22]}$

Received: November 12, 1998 [Z12656 IE] German version: Angew. Chem. 1999, 111, 1324-1327

Keywords: bioorganic chemistry - enzyme inhibitors • molecular recognition $\cdot$ nucleotides $\cdot$ trisphosphonic acids

[1] A. G. McLennan, P. C. Zamecnik in $A p_{4} A$ and Other Dinucleoside Polyphosphates (Ed.: A. G. McLennan), CRC Press, Boca Raton, FL, 1992, pp. $1-7$.

[2] a) Z. Siprashvili, G. Sozzi, L. D. Barnes, P. McCue, A. K. Robinson, V. Eryomin, L. Sard, E. Ragliabue, A. Graco, L. Fusetti, G. Schwartz, M. A. Pierotti, C. M. Croce, K. Huebner, Proc. Natl. Acad. Sci. USA 1997, 94, 13771 - 13776; b) L. D. Barnes, P. N. Garrison, Z. Siprashvili, A. Guranowski, A. K. Robinson, S. W. Ingram, C. M. Croce, M. Ohta, K. Huebner, Biochemistry 1996, 35, 11529-11535.

[3] a) C. Brenner, H. C. Pace, P. N. Garrison, A. K. Robinson, A. Rösler, A. Liu, G. M. Blackburn, C. M. Croce, K. Huebner, L. D. Barnes, Protein Eng. 1997, 10, 1461 -1463; b) H. C. Pace, P. N. Garrison, A. K. Robinson, L. D. Barnes, A. Draganescu, A. Rösler, G. M. Blackburn, Z. Siprashvili, C. M. Croce, K. Huebner, C. Brenner, Proc. Natl. Acad. Sci. USA 1998, 95, 5484-5489.

[4] D. Maksel, A. Guranowski, S. C. Ilgoutz, A. Moir, G. M. Blackburn, K. R. Gayler, Biochem. J. 1998, 329, 313-319, and references therein.

[5] A. Guranowski, P. Brown, P. A. Ashton, G. M. Blackburn, Biochemistry 1994, 33, $235-240$.

[6] G. M. Blackburn, M.-J. Guo, A. G. McLennan in $A p_{4} A$ and Other Dinucleoside Polyphosphates (Ed.: A. G. McLennan), CRC Press, Boca Raton, FL, 1992, pp. 305-342.

[7] D. M. Williams, D. L. Jakeman, J. S. Vyle, M. P. Williamson, G. M. Blackburn, Collect. Czech. Chem. Commun. 1996, 31, 88-91; D. M. Williams, D. L. Jakeman, J. S. Vyle, M. P. Williamson, G. M. Blackburn, Bioorg. Med. Chem. Lett. 1998, 8, 2603-2608.

[8] G. M. Blackburn, Chem. Ind. (London) 1981 (5), 134-138.

[9] G. E. Lienhard, I. I. Secemski, J. Biol. Chem. 1973, 248, 1121-1127.

[10] R. M. Dixon, G. Lowe, J. Biol. Chem. 1989, 264, 2069-2074.

[11] X. Liu, X.-R. Zhang, G. M. Blackburn, Chem. Commun. 1997, 52, 87 88.

[12] a) C. Brenner, P. Garrison, J. Gilmour, D. Peisach, D. Ringe, G. A. Petsko, J. M. Lowenstein, Nature Struct. Biol. 1997, 4, 231-238; b) M. J. Bessman, D. N. Frick, S. F. O'Handley, J. Biol. Chem. 1996, 271, 25059-25062; c) G. Sozzi, K. Huebner, C. M. Croce, Adv. Cancer Res. 1998, 74, $141-166$.

[13] H. Gross, B. Costisella, I. Keitel, S. Ozegowski, Phosphorus Sulfur Silicon 1993, 83, 203-207.

[14] X. Liu, H. Adams, G. M. Blackburn, Chem. Commun. 1998, 2619 2620.

[15] V. J. Davisson, D. R. Davis, V. M. Dixit, C. D. Poulter, J. Org. Chem. 1987, 52, 1794-1801

[16] J. G. Moffatt, H. G. Khorana, J. Am. Chem. Soc. 1961, 83, 649-658.

[17] See V. Wittmann, C. H. Wong, J. Org. Chem. 1997, 62, 2144-2147.

[18] These tripodal analogues have been fully characterized by NMR spectroscopy and mass spectrometry along with all other nucleotide analogues described here. Positive-ion HR-MS: 2: $\mathrm{m} / z$ 571.971, calcd for $\left[\mathrm{C}_{11} \mathrm{H}_{15} \mathrm{~N}_{5} \mathrm{O}_{12} \mathrm{P}_{3} \mathrm{Na}_{3}+\mathrm{H}\right]^{+}: 571.970 ; 4: \mathrm{m} / z$ 1080.898, calcd for $\left[\mathrm{C}_{21} \mathrm{H}_{24} \mathrm{ClN}_{10} \mathrm{O}_{21} \mathrm{P}_{5} \mathrm{Na}_{6}+\mathrm{H}\right]^{+}: 1080.896\left({ }^{35} \mathrm{Cl}\right) ; \mathbf{6}: \mathrm{m} / \mathrm{z}$ 1398.985, calcd for $\left[\mathrm{C}_{31} \mathrm{H}_{38} \mathrm{~N}_{15} \mathrm{O}_{27} \mathrm{P}_{6} \mathrm{Na}_{6}+\mathrm{Na}\right]^{+}$: 1398.977; 8: 1296.023, calcd for $\left[\mathrm{C}_{31} \mathrm{H}_{37} \mathrm{~N}_{15} \mathrm{O}_{24} \mathrm{P}_{5} \mathrm{Na}_{5}+\mathrm{H}\right]^{+}$: 1296.021 . Their purity was established as $98 \%$ or greater by HPLC (BioRad MA70 anion exchange column, $50 \mathrm{~mm} \rightarrow 700 \mathrm{~mm} \mathrm{NH}_{4}{ }^{+} \mathrm{HCO}_{3}^{-}$gradient elution).

[19] A. Guranowski, A. Sillero in $A p_{4} A$ and Other Dinucleoside Polyphosphates (Ed.: A. G. McLennan), CRC Press, Boca Raton, FL, 1992, pp. $81-133$.
[20] N. M. H. Thorne, S. Hankin, M. C. Wilkinson, C. Nunez, R. Barraclough, A. G. McLennan, Biochem. J. 1995, 311, 717-721.

[21] A. Guranowski, E. Starzynska, P. Brown, G. M. Blackburn, Biochem. J. 1997, 328, 257-262.

[22] A. Guranowski, E. Starzynska, E. Bojarska, J. Stepinski, E. Darzynkiewicz, Protein Expression Purif. 1996, 8, 416-422.

[23] H. Jakubowski, A. Guranowski, J. Biol. Chem. 1983, 258, $9982-9989$.

\section{Highly Regio- and Stereoselective Synthesis of Mannose-Containing Oligosaccharides with Acetobromo Sugars as the Donors and Partially Protected Mannose Derivatives as the Acceptors via Sugar Orthoester Intermediates $* *$}

\section{Wei Wang and Fanzuo Kong*}

Many biologically important natural products such as glycoproteins,${ }^{[1]}$ the ubiquitous components of the cell membrane, contain an oligomannopyranose core, while the cell wall of yeast contains branched mannans. ${ }^{[2]}$ Mannose-containing oligosaccharides have been synthesized by wellestablished methods ${ }^{[3]}$ that involve multistep selective protection and deprotection procedures. The use of unprotected or partially protected mannose and acetobromo sugars as raw materials in glycosylations is very attractive for organic chemists because the synthetic routes can be substantially simplified. In previous work ${ }^{[4 a]}$ we described a new method for regio- and stereoselective synthesis of oligosaccharides by an orthoester $^{[4 \mathrm{~d}-\mathrm{m}]}$ formation/rearrangement procedure with unprotected glucopyranosides as the glycosyl acceptors and acetobromo sugars as the donors which gave $1 \rightarrow 6$-linked oligosaccharides in satisfactory yields. In addition, 3-selective glycosylation was achieved with partially protected glucose acceptors containing unprotected 2,3- or 3,4-hydroxy groups. It was found, however, that the glycosylation with unprotected glycosides as the acceptors was rather slow and difficult to monitor owing to the poor solubility of the acceptors in the reaction media. We now report a new strategy for highly regio- and stereoselective synthesis of mannose-containing diand oligosaccharides via orthoester intermediates by coupling acetobromo sugars with partially protected mannose derivatives as the acceptors, in particular naked mannose 1,2-Oethylidenate.

It is well known that 3,6-branched mannotrisaccharide Manp $\alpha 1 \rightarrow 6(\operatorname{Manp} \alpha 1 \rightarrow 3)$ Man is present in all asparagine-

[*] Prof. Dr. F. Kong, Dr. W. Wang

Research Center for Eco-Environmental Sciences

Academia Sinica

P. O. Box 2871, Beijing 100085 (China)

Fax: $(+86) 10-62923563$

E-mail: fzkong@mail.rcees.ac.cn

[**] This work was supported by the Chinese Academy of Sciences (Project KJ952 $\mathrm{J}_{1} 510$ ) and the National Natural Science Foundation of China (Project 29802009).

Supporting information for this article is available on the WWW under http://www.wiley-vch.de/home/angewandte/ or from the author. 
linked oligosaccharides, and it is the major Con A binding epitope on oligomannose-type carbohydrates. ${ }^{[5]}$ The syntheses of the trisaccharide involve the use of different protective groups and lengthy reaction routes leading to the product in low yields. ${ }^{[6]}$ With the newly developed orthoester formation/rearrangement strategy the trisaccharide was readily synthesized ${ }^{[7]}$ by coupling $2,3,4,6-$ tetra- $O$-acetyl- $\alpha$-D-mannopyranosyl bromide (1, 2.4 equiv) with 1,2- $O$-ethylidene- $\beta$-D-mannopyranose (2, 1 equiv) in the presence of silver trifluoromethanesulfonate (AgOTf, 2.2 equiv) and 2,4-lutidine ( 2.4 equiv) followed by rearrangement (Scheme 1).

The formation of trisaccharide diorthoester $\mathbf{3}$ is highly regioselective; no 4-substitution was found, and $\mathbf{3}$ and $\mathbf{4}$ were obtained as white crystals. The structure of $\mathbf{3}$ was unambiguously verified by its ${ }^{1} \mathrm{H}$ NMR spectrum, ${ }^{[8]}$ which showed two characteristic signals at $\delta=5.54$ and 5.50 for $\mathrm{H}^{\prime}$ and $\mathrm{H}^{\prime \prime}$ respectively, and by its acetylation product 4 , which exhibited a new triplet at $\delta=5.12$, a clear indication ${ }^{[8]}$ of 3,6 branched glycosylation. Rearrangement ${ }^{[4 \mathrm{a}-\mathrm{c}, 9]}$ of $\mathbf{4}$ in the presence of trimethylsilyl trifluoromethanesulfonate (TMSOTf) gave the 3,6-branched mannotrisaccharide $\mathbf{5}$ in high yield. Its 2D ${ }^{1} \mathrm{H}$ NMR spectrum also gave a clear indication ${ }^{[8]}$ of 3,6-branched glycosylation.

The orthoester $\mathbf{3}$ is a very important intermediate. It can be modified at the reducing ends to couple sugar donors at the $2^{\prime}$ - and $2^{\prime \prime}$ positions, and its transformation to 5 followed by removal of the 1,2-ethylidene group can lead to suitable glycosyl donors such as the Schmidt reagent and phenyl thioglycoside. Subsequent coupling with sugar acceptors affords $\alpha_{-}^{[3 c]}$ and $\beta$ linked $^{[10]}$ oligosaccharides respectively.

Encouraged by the success in coupling $\mathbf{2}$ with $\mathbf{1}$, we tried the glycosylation of $\mathbf{2}$ with disaccharide as the glycosyl donor. Thus, acetobromolactose (2.2 equiv) was coupled with 2 (1 equiv) under the same conditions as described for the prepara-

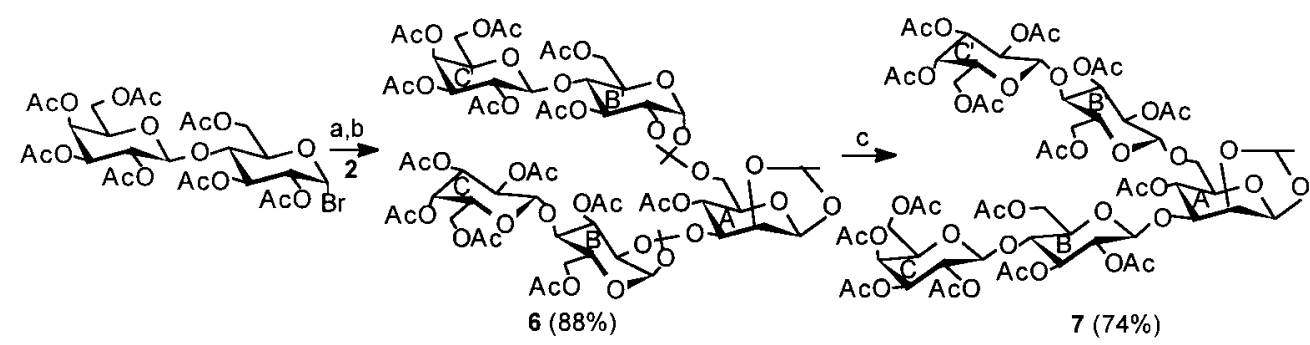

Scheme 2. Conditions and reagents: a) AgOTf/2,4-lutidine, $\mathrm{CH}_{2} \mathrm{Cl}_{2}$, molecular sieves (4 ̊), 4 h; b) Ac $\mathrm{O} /$ anhydrous pyridine; c) TMSOTf $/ \mathrm{CH}_{2} \mathrm{Cl}_{2}$, molecular sieves (4 ̊̊), $\mathrm{N}_{2}, 30 \mathrm{~min}$.

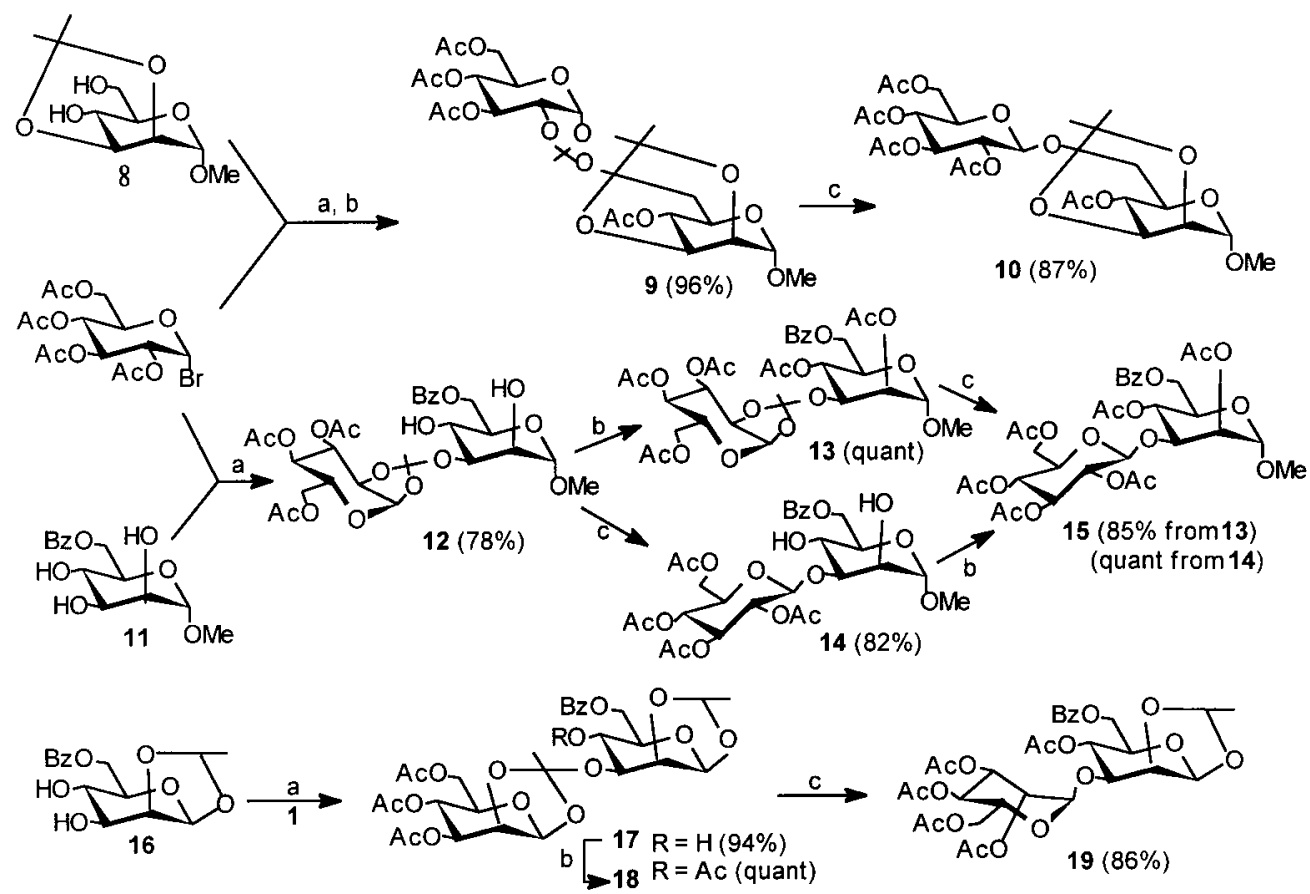

Scheme 3. Conditions and reagents: a) AgOTf/2,4-lutidine, $\mathrm{CH}_{2} \mathrm{Cl}_{2}$, molecular sieves (4 ̊), 2-3 h; b) Ac $\mathrm{O} /$ anhydrous pyridine; c) TMSOTf/ $\mathrm{CH}_{2} \mathrm{Cl}_{2}$, molecular sieves (4 ̊̊), $\mathrm{N}_{2}, 30-40$ min. 
6-linked orthoester was formed as the sole product in almost quantitative yield. Its acetylation to 9 followed by rearrangement readily furnished $\mathbf{1 0}$. With methyl 6- $O$-benzoyl- $\alpha$-Dmannopyranoside $^{[11]}(\mathbf{1 1})$ as the acceptor, the 3-linked orthoester $\mathbf{1 2}$ was obtained in a high yield. Its acetylation to $\mathbf{1 3}$ followed by rearrangement, or its rearrangement to $\mathbf{1 4}$ followed by acetylation produced the same compound $\mathbf{1 5}$, indicating that both orthoester formation and rearrangement were regio- and stereoselective. The same 3-selectivity was obtained with 6 - $O$-benzoyl-1,2- $O$-ethylidene- $\beta$-D-mannopyranose (16) as the acceptor, when the 1,3-linked disaccharide 19 was formed in a satisfactory yield.

Since the rearrangement of $\mathbf{1 2}$ to $\mathbf{1 4}(82 \%)$ and the disaccharides in similar yields, and the rearrangement of $\mathbf{4}$ afforded the trisaccharide $\mathbf{5}$, also in a high yield, we rationalized the mechanism of the rearrangement as shown in Scheme 4. When TMSOTf was added to the solution of $\mathbf{1 2}$ in dichloromethane, selective bond cleavage of the orthoester linkage rather than 2- or 4-OH bond cleavage occurred to give a closely associated ion pair (form $\mathbf{A}$ ). When this ion pair reaches an appropriate six-membered ring geometry (form $\mathbf{B}$ ) rearrangement occurs to afford the required disaccharide $\mathbf{1 4}$. We still consider that steric factors are mainly responsible for the high regioselectivity in orthoester formation. ${ }^{[4]}$ rearrangement of $\mathbf{1 3}$ to $\mathbf{1 5}(85 \%)$ afforded the respective

New York, 1996, 125; c) F. Barresi, O. Hindsgaul, J. Carbohydr. Chem. 1995, 14, 1043; d) R. R. Schmidt, W. Kinzy, Adv. Carbohydr. Chem. Biochem. 1994, 50, 21; e) K. Toshima, K. Tatsuta, Chem. Rev. 1993, 93 , 1503. Glycosylsulfoxide donors: L. Yan, D. Kahne, J. Am. Chem. Soc. 1996, 118, 9239. 1-Hydroxyglycosyl donors: B. A. Garcia, J. A. Poole, D. Y. Gin, J. Am. Chem. Soc. 1997, 119, 7597. Glycal donors: S. J. Danishefsky, M. T. Bilodeau, Angew. Chem. 1996, 108, 1482; Angew. Chem. Int. Ed. Engl. 1996, 35, 1380. 1,2-Anhydroglycosyl donors: Y. Du, F. Kong, Tetrahedron Lett. 1995, 427.

[4] a) W. Wang, F. Kong, J. Org. Chem. 1998, 63, 5744; b) F. Kong, W. Wang, Chinese Pat. Appl. 971257788.4; c) F. Kong, W. Wang, Chinese Pat. Appl. 98103242.7; d) B. Ernst, A. De Mesmaeker, B. Wagner, T. Winkler, Tetrahedron Lett. 1990, 6167; e) J. Banoub, P. Boullanger, M. Potier, G. Descotes, Tetrahedron Lett. 1986, 4145; f) B. M. Dahlin, P. J. Garegg, R. Johansson, B. Samuelsson, U. Orn, Acta Chem. Scand. Ser. $B$ 1981, 35, 669; g) J. Banoub, D. R. Bundle, Can. J. Chem. 1979, 57, 2091; h) T. Ogawa, M. Matsui, Carbohydr. Res. 1976, 51, C13; i) S. E. Zurabyan, M. M. Tikhomirov, V. A. Nesmeyanov, A. Y. Khorlin, Carbohydr. Res. 1973, 26, 117; j) G. Wulff, W. Kruger, Carbohydr. Res. 1971, 19, 139; k) N. K. Kochetkov, A. F. Bochkov, T. A. Sokolovskaya, V. J. Snyatkova, Carbohydr. Res. 1971, 16, 17; 1) N. K. Kochetkov, A. J. Khorlin, A. F. Bochkov, Tetrahedron 1967, 23, 693.

[5] a) D. K. Mandal, L. Bhattacharyya, S. H. Koenig, R. D. Brown, S. Oscarson, C. F. Brewer, Biochemistry 1994, 33, 1157; b) D. K. Mandal, C. F. Brewer, Biochemistry 1992, 32, 5116; c) B. A. Williams, M. C. Chervenak, E. J. Toone, J. Biol. Chem. 1992, 267, 22907; d) M. C. Chervenak, E. J. Toone, J. Am. Chem. Soc. 1994, 116, 10533.

[6] a) M. W. Francoise, J. R. Brisson, J. P. Carver, J. J. Krepinsky, Carbohydr. Res. 1982, 103, 15; b) T. Ogawa, K. Katano, M. Matsui, Carbohydr. Res. 1978, 64, C3; c) J. Arnarp, J. Lonngren, Acta Chem. Scand. Ser. B 1978, 32, 696.

[7] Typical conditions for orthoester preparation: To a stirred solution of 2,3,4,6-tetra- $O$-acetyl- $\alpha$-D-mannopyranosyl bromide $(\mathbf{1}, 1.02 \mathrm{~g}, 2.5 \mathrm{mmol}), 2,4$-lutidine $(276 \mu \mathrm{L}, 2.4 \mathrm{mmol})$, and $1,2-O$-ethylidene- $(R, S)-\beta$-Dmannopyranose $(\mathbf{2}, 206 \mathrm{mg}, 1 \mathrm{mmol})$ in dichloromethane $(20 \mathrm{~mL})$ under nitrogen atmosphere was added $\mathrm{AgOTf}(565 \mathrm{mg}, 2.2 \mathrm{mmol})$ in a dark room. The reaction was carried out at room temperature and monitored by TLC (petroleum ether/ethyl acetate, 1/1.5). After completion of the reaction the mixture was partitioned between dichloromethane and water, the organic phase

Scheme 4 .

In summary, here we present a very effective regio- and stereoselective glycosylation method with 1,2 - $O$-ethylidenated mannose or partially protected mannosides as glycosyl acceptors and simple acetobromo sugars as the glycosyl donors by an orthoester formation/rearrangement procedure. A number of $1 \rightarrow 6$-, $1 \rightarrow 3$-, and 3,6-branched oligosaccharides with exclusive 1,2-trans linkage were readily synthesized by the new strategy. This approach, in terms of yields, simplicity, and efficiency, will be a general one for the synthesis of mannose-containing oligosaccharides.

Received: November 10, 1998 [Z12648 IE] German version: Angew. Chem. 1999, 111, 1330-1333

Keywords: oligosaccharides $\cdot$ protecting groups $\cdot$ rearrangements $\cdot$ synthetic methods

[1] A. Kobata in Biology of Carbohydrates, Vol. 2 (Ed.: V. Ginsburg), IRL Press, New York, 1980, pp. 87-161.

[2] a) T. Kanbe, J. E. Cutler, Infect. Immun. 1994, 62, 1662; b) M. Stratford, Yeast 1992, 8, 635; c) R. D. Nelson, N. Shibata, R. P. Podzorski, M. J. Herron, Clin. Microbiol. Rev. 1991, 4, 1; d) R. P. Podzorski, G. R. Gray, R. D. Nelson, J. Immunol. 1990, 144, 707.

[3] General reviews: a) S. Hanessian, Preparative Carbohydrate Chemistry, Marcel Dekker, New York, 1997, Chapters 12-22; b) S. H. Khan, R. A. O'Neil, Modern Methods in Carbohydrate Synthesis, Harwood, was concentrated, dried, and subjected to column chromatography on silica gel with petroleum ether/ethyl acetate $(1 / 1)$ as the eluent to give the trisaccharide diorthoester $\mathbf{3}$ (91\% yield based on acceptor 2 ) as the sole product. The procedure for the preparation of the disaccharide orthoesters is the same as described above except for the amounts of the acetoglycobromide donors $(\approx 1.2$ equiv $)$ and the promoters $(\approx 1.2$ equiv). Typical rearrangement conditions: To a stirred solution of sugar-sugar orthoester $4(580 \mathrm{mg}, 0.64 \mathrm{mmol})$ in dichloromethane $(10 \mathrm{~mL})$ was added TMSOTf $(12 \mu \mathrm{L}, 0.1$ equiv) under nitrogen atmosphere and the reaction was monitored by TLC (petroleum ether/ethyl acetate, 1/1). After completion of the reaction the mixture was treated with triethylamine $(20 \mu \mathrm{L})$, filtered, and the filtrate was washed with $\mathrm{CH}_{2} \mathrm{Cl}_{2}$. The combined solution was washed with $1 \mathrm{M} \mathrm{HCl}$ $(10 \mathrm{~mL})$, saturated aqueous $\mathrm{NaHCO}_{3}(10 \mathrm{~mL})$, and aqueous $\mathrm{NaCl}$ $(2 \times 10 \mathrm{~mL})$, dried over anhydrous $\mathrm{Na}_{2} \mathrm{SO}_{4}$, and concentrated. The residue was subjected to column chromatography on silica gel with petroleum ether/ethyl acetate $(1 / 1)$ as the eluent to give $\mathbf{5}(510 \mathrm{mg}$, $88 \%$ ).

[8] Physical data and ${ }^{1} \mathrm{H}$ NMR (400 MHz, $\mathrm{CDCl}_{3}, 25^{\circ} \mathrm{C}$, TMS) data for ${ }^{1} \mathrm{H}$ NMR: $\delta=5.54,5.50\left(2 \mathrm{~d},{ }^{3} J\left(\mathrm{H}_{1^{\prime}\left(1^{\prime \prime}\right)}, \mathrm{H}_{2^{\prime}\left(2^{\prime \prime}\right)}\right)=2.7 \mathrm{~Hz}, 2 \mathrm{H} ; \mathrm{H}-1^{\prime}, 1^{\prime \prime}\right)$, $5.30-5.15$ (m, H-1, 3', 3", 4', 4", 6 H; $\left.\mathrm{CH}_{3} \mathrm{CH}\right), 4.75,4.63$ (2 dd, ${ }^{3} J$ $\left.\left(\mathrm{H}_{2^{\prime}}, \mathrm{H}_{3^{\prime}}\right)=3.8 \mathrm{~Hz},{ }^{3} J\left(\mathrm{H}_{2^{\prime \prime}}, \mathrm{H}_{3^{\prime \prime}}\right)=3.9 \mathrm{~Hz}, 2 \mathrm{H} ; \mathrm{H}-2^{\prime}, 2^{\prime \prime}\right), 4.23-4.13(\mathrm{~m}$, $\left.4 \mathrm{H} ; \mathrm{H}-6^{\prime}, 6^{\prime \prime}\right), 4.06\left(\mathrm{dd},{ }^{3} J\left(\mathrm{H}_{1}, \mathrm{H}_{2}\right)=2.4 \mathrm{~Hz},{ }^{3} J\left(\mathrm{H}_{2}, \mathrm{H}_{3}\right)=3.6 \mathrm{~Hz}, 1 \mathrm{H}\right.$; H-2), $3.84-3.68$ (m, 6H; H-3, 4, 5', 5', 6), 3.40-3.35 (m, 1H; H-5), 2.11, 2.10, 2.07, 2.07, 2.06, $2.05\left(6 \mathrm{~s}, 18 \mathrm{H} ; 6 \mathrm{CH}_{3} \mathrm{CO}\right), 1.85,1.74(2 \mathrm{~s}, 6 \mathrm{H}$; $\left.2 \mathrm{CH}_{3} \mathrm{CO}\right), 1.47\left(\mathrm{~d},{ }^{3} \mathrm{~J}(\mathrm{H}, \mathrm{H})=5.0 \mathrm{~Hz}, 3 \mathrm{H} ; \mathrm{CH}_{3} \mathrm{CH}\right)$. 4: m.p. $135-$ $138^{\circ} \mathrm{C} ;[\alpha]_{\mathrm{D}}=+0.4(c=0.1$, trichloromethane $) ;{ }^{1} \mathrm{H}$ NMR: $\delta=5.49$, $5.43\left(2 \mathrm{~d},{ }^{3} J\left(\mathrm{H}_{1^{\prime}}, \mathrm{H}_{2^{\prime}}\right),{ }^{3} J\left(\mathrm{H}_{1^{\prime \prime}}, \mathrm{H}_{2^{\prime \prime}}\right)=2.8 \mathrm{~Hz}, 2.6 \mathrm{~Hz}, 2 \mathrm{H} ; \mathrm{H}-1^{\prime}, 1^{\prime \prime}\right)$, $5.30-5.16\left(\mathrm{~m}, 6 \mathrm{H} ; \mathrm{H}-1,3^{\prime}, 3^{\prime \prime}, 4^{\prime}, 4^{\prime \prime}, \mathrm{CH}_{3} \mathrm{CH}\right), 5.12\left(\mathrm{t},{ }^{3} J(\mathrm{H}, \mathrm{H})=\right.$ 3-6. 3: m.p. $110-112^{\circ} \mathrm{C} ;[\alpha]_{\mathrm{D}}=+5.2(c=1.5$ trichloromethane); 
$9.6 \mathrm{~Hz}, 1 \mathrm{H} ; \mathrm{H}-4), 4.66\left(\mathrm{dd},{ }^{3} J\left(\mathrm{H}_{1^{\prime \prime}}, \mathrm{H}_{2^{\prime \prime}}\right)=2.9 \mathrm{~Hz},{ }^{3} J\left(\mathrm{H}_{2^{\prime \prime}}, \mathrm{H}_{3^{\prime \prime}}\right)=3.9 \mathrm{~Hz}\right.$, $1 \mathrm{H}$; H-2 $\left.2^{\prime \prime}\right), 4.58\left(\mathrm{dd},{ }^{3} J\left(\mathrm{H}_{1^{\prime}}, \mathrm{H}_{2^{\prime}}\right)=2.8 \mathrm{~Hz},{ }^{3} J\left(\mathrm{H}_{2^{\prime}} \mathrm{H}_{3^{\prime}}\right)=4.0 \mathrm{~Hz}, 1 \mathrm{H}\right.$; $\left.\mathrm{H}-2^{\prime}\right), 4.24-4.08\left(\mathrm{~m}, 5 \mathrm{H} ; \mathrm{H}-2,6^{\prime}, 6^{\prime \prime}\right), 4.01\left(\mathrm{dd},{ }^{3} \mathrm{~J}\left(\mathrm{H}_{2}, \mathrm{H}_{3}\right)=4.6 \mathrm{~Hz},{ }^{3} J\right.$ $\left.\left(\mathrm{H}_{3}, \mathrm{H}_{4}\right)=9.6 \mathrm{~Hz}, 1 \mathrm{H} ; \mathrm{H}-3\right), 3.75-3.65\left(\mathrm{~m}, 2 \mathrm{H} ; \mathrm{H}-5^{\prime}, 5^{\prime \prime}\right), 3.60\left(\mathrm{~d},{ }^{2} J\right.$ $\left.\left(\mathrm{H}_{6 \mathrm{a}}, \mathrm{H}_{6 \mathrm{~b}}\right)=4.5 \mathrm{~Hz}, 2 \mathrm{H} ; \mathrm{H}-6\right), 3.55-3.50(\mathrm{~m}, 1 \mathrm{H}, \mathrm{H}-5), 2.12,2.10,2.07$, 2.06, 2.06, 2.05, $2.04\left(7 \mathrm{~s}, 21 \mathrm{H} ; 7 \mathrm{CH}_{3} \mathrm{CO}\right), 1.77,1.71(2 \mathrm{~s}, 6 \mathrm{H} ; 2$ $\left.\mathrm{CH}_{3} \mathrm{CO}_{3}\right), 1.49\left(\mathrm{~d},{ }^{3} \mathrm{~J}(\mathrm{H}, \mathrm{H})=4.9 \mathrm{~Hz}, 3 \mathrm{H} ; \mathrm{CH}_{3} \mathrm{CH}\right)$. 5: m.p. $177-$ $178^{\circ} \mathrm{C} ;[\alpha]_{\mathrm{D}}=+16.9(c=0.15$, trichloromethane $) ;{ }^{1} \mathrm{H}$ NMR: $\delta=$ $5.36-5.21$ (m, 8H; H-1, 2', 3', 3", 4, 4', 4", $\left.\mathrm{CH}_{3} \mathrm{CH}\right), 5.14$ (dd, ${ }^{3} J$ $\left.\left(\mathrm{H}_{1^{\prime \prime}}, \mathrm{H}_{2^{\prime \prime}}\right)=1.9 \mathrm{~Hz},{ }^{3} J\left(\mathrm{H}_{2^{\prime \prime}}, \mathrm{H}_{3^{\prime \prime}}\right)=3.1 \mathrm{~Hz}, 1 \mathrm{H} ; \mathrm{H}-2^{\prime \prime}\right), 4.98\left(\mathrm{~d},{ }^{3} J\right.$ $\left.\left(\mathrm{H}_{1^{\prime \prime}}, \mathrm{H}_{2^{\prime \prime}}\right)=1.9 \mathrm{~Hz}, 1 \mathrm{H} ; \mathrm{H}-1^{\prime \prime}\right), 4.78\left(\mathrm{~d},{ }^{3} J\left(\mathrm{H}_{1^{\prime}}, \mathrm{H}_{2^{\prime}}\right)=1.7 \mathrm{~Hz}, 1 \mathrm{H}\right.$; H-1'), $4.34-4.27$ (m, 4H; H-2, 5', 6"), $4.10-4.01$ (m, 3H; H-5", 6' ), 3.91 $\left(\mathrm{dd},{ }^{3} J\left(\mathrm{H}_{2}, \mathrm{H}_{3}\right)=3.8 \mathrm{~Hz},{ }^{3} J\left(\mathrm{H}_{3}, \mathrm{H}_{4}\right)=9.7 \mathrm{~Hz}, 1 \mathrm{H} ; \mathrm{H}-3\right), 3.80\left(\mathrm{dd},{ }^{3} J\right.$ $\left.\left(\mathrm{H}_{5}, \mathrm{H}_{6 \mathrm{a}}\right)=6.3 \mathrm{~Hz},{ }^{2} J\left(\mathrm{H}_{6 \mathrm{a}}, \mathrm{H}_{6 \mathrm{~b}}\right)=10.4 \mathrm{~Hz}, 1 \mathrm{H} ; \mathrm{H}-6_{\mathrm{a}}\right), 3.62-3.56(\mathrm{~m}$, $\left.2 \mathrm{H} ; \mathrm{H}-5,6_{\mathrm{b}}\right), 2.16,2.15,2.15,2.11,2.09,2.06,2.06,2.00,1.98(9 \mathrm{~s}, 27 \mathrm{H} ; 9$ $\left.\mathrm{CH}_{3} \mathrm{CO}\right), 1.53\left(\mathrm{~d},{ }^{3} \mathrm{~J}(\mathrm{H}, \mathrm{H})=5.0 \mathrm{~Hz}, 3 \mathrm{H} ; \mathrm{CH}_{3} \mathrm{CH}\right) .6$ : $[\alpha]_{\mathrm{D}}=+2.4(c=$ 0.15 , trichloromethane); ${ }^{1} \mathrm{H}$ NMR: $\delta=5.69\left(\mathrm{~d},{ }^{3} J\left(\mathrm{H}_{1 \mathrm{~B}}, \mathrm{H}_{2 \mathrm{~B}}\right)=5.1 \mathrm{~Hz}\right.$, $1 \mathrm{H} ; \mathrm{H}-1 \mathrm{~B}), 5.65\left(\mathrm{~d},{ }^{3} \mathrm{~J}\left(\mathrm{H}_{1 \mathrm{~B}^{\prime}}, \mathrm{H}_{2 \mathrm{~B}^{\prime}}\right)=5.2 \mathrm{~Hz}, 1 \mathrm{H} ; \mathrm{H}-1 \mathrm{~B}^{\prime}\right), 5.50\left(\mathrm{dd},{ }^{3} J\right.$ $\left.\left(\mathrm{H}_{2 \mathrm{~B}}, \mathrm{H}_{3 \mathrm{~B}}\right)=2.4 \mathrm{~Hz},{ }^{3} J\left(\mathrm{H}_{3 \mathrm{~B}}, \mathrm{H}_{4 \mathrm{~B}}\right)=1.2 \mathrm{~Hz}, 1 \mathrm{H} ; \mathrm{H}-3 \mathrm{~B}\right), 5.42\left(\mathrm{t},{ }^{3} J\right.$ $\left.(\mathrm{H}, \mathrm{H})=1.9 \mathrm{~Hz}, 1 \mathrm{H} ; \mathrm{H}-3 \mathrm{~B}^{\prime}\right), 5.38\left(2 \mathrm{~d},{ }^{3} \mathrm{~J}(\mathrm{H}, \mathrm{H})=3.3 \mathrm{~Hz}, 2 \mathrm{H} ; \mathrm{H}-4 \mathrm{C}\right.$, $\left.4 \mathrm{C}^{\prime}\right), 5.26\left(\mathrm{~d},{ }^{3} J\left(\mathrm{H}_{1 \mathrm{~A}}, \mathrm{H}_{2 \mathrm{~A}}\right)=1.6 \mathrm{~Hz}, 1 \mathrm{H} ; \mathrm{H}-1 \mathrm{~A}\right), 5.26\left(\mathrm{q},{ }^{3} J(\mathrm{H}, \mathrm{H})=\right.$ $\left.5.0 \mathrm{~Hz}, 1 \mathrm{H} ; \mathrm{CH}_{3} \mathrm{CH}\right), 5.16\left(2 \mathrm{dd},{ }^{3} J\left(\mathrm{H}_{1 \mathrm{C}\left(\mathrm{C}^{\prime}\right)}, \mathrm{H}_{2 \mathrm{C}\left(\mathrm{C}^{\prime}\right)}\right)=8.0 \mathrm{~Hz},{ }^{3} J\right.$ $\left.\left(\mathrm{H}_{2 \mathrm{C}\left(\mathrm{C}^{\prime}\right)}, \mathrm{H}_{3 \mathrm{C}\left(\mathrm{C}^{\prime}\right)}\right)=9.6 \mathrm{~Hz}, 2 \mathrm{H} ; \mathrm{H}-2 \mathrm{C}, 2 \mathrm{C}^{\prime}\right), 5.10\left(\mathrm{t},{ }^{3} \mathrm{~J}(\mathrm{H}, \mathrm{H})=9.5 \mathrm{~Hz}\right.$, $1 \mathrm{H} ; \mathrm{H}-4 \mathrm{~A}), 5.00\left(2 \mathrm{dd}, 2 \mathrm{H} ; \mathrm{H}-3 \mathrm{C}, 3 \mathrm{C}^{\prime}\right), 4.60,4.55\left(2 \mathrm{~d},{ }^{3} J\right.$ $\left.\left(\mathrm{H}_{1 \mathrm{C}\left(\mathrm{C}^{\prime}\right)}, \mathrm{H}_{2 \mathrm{C}\left(\mathrm{C}^{\prime}\right)}\right)=8.0 \mathrm{~Hz}, 2 \mathrm{H} ; \mathrm{H}-1 \mathrm{C}, 1 \mathrm{C}^{\prime}\right), 4.41(\mathrm{dd}, 1 \mathrm{H} ; \mathrm{H}-2 \mathrm{~B}), 4.35$ (dd, 1H; H-2B'), 4.30-4.20 (m, 3H; H-2A, 6B $\left.\mathrm{B}_{\mathrm{a}}, 6 \mathrm{~B}_{\mathrm{a}}^{\prime}\right), 4.13-4.05(\mathrm{~m}$, $\left.6 \mathrm{H} ; \mathrm{H}-6 \mathrm{~B}_{\mathrm{b}}, 6 \mathrm{~B}_{\mathrm{b}}^{\prime}, 6 \mathrm{C}, 6 \mathrm{C}^{\prime}\right), 3.98-3.88\left(\mathrm{~m}, 3 \mathrm{H} ; \mathrm{H}-3 \mathrm{~A}, 5 \mathrm{C}, 5 \mathrm{C}^{\prime}\right), 3.85-$ $3.78\left(\mathrm{~m}, 2 \mathrm{H} ; \mathrm{H}-5 \mathrm{~B}, 5 \mathrm{~B}^{\prime}\right), 3.64\left(2 \mathrm{t},{ }^{3} J(\mathrm{H}, \mathrm{H})=9.5 \mathrm{~Hz}, 2 \mathrm{H} ; \mathrm{H}-4 \mathrm{~B}, 4 \mathrm{~B}^{\prime}\right)$, $3.60-3.58$ (m, 3 H; H-5A, 6A), 2.17-1.98 (13 s, 39H; $\left.13 \mathrm{CH}_{3} \mathrm{CO}\right), 1.74$, $1.68\left(2 \mathrm{~s}, 6 \mathrm{H} ; 2 \mathrm{CH}_{3} \mathrm{CO}_{3}\right), 1.47\left(\mathrm{~d},{ }^{3} \mathrm{~J}(\mathrm{H}, \mathrm{H})=5.0 \mathrm{~Hz}, 3 \mathrm{H} ; \mathrm{CH}_{3} \mathrm{CH}\right)$.

[9] a) J. Gass, M. Strobl, P. Kosma, Carbohydr. Res. 1993, 244, 69; b) M. L. Sznaidman, S. C. Johnson, C. Crasto, S. M. Hecht, J. Org. Chem. 1995, 60, 3942; c) T. Ogawa, K. Beppu, S. Nakabayashi, Carbohydr. Res. 1981, 93, C6; d) W. Wang, F. Kong, Tetrahedron Lett. 1998, 1937.

[10] D. Crich, S. Sun, J. Am. Chem. Soc. 1998, 120, 435.

[11] J. Xia, Y. Hui, Synth. Commun. 1995, 25, 2235.

\section{Chemoenzymatic Synthesis of a Characteristic Glycophosphopeptide from the Transactivation Domain of the Serum Response Factor**}

\section{Jörg Sander and Herbert Waldmann*}

Phosphorylation $^{[1]}$ and O-glycosidic attachment of $\mathrm{N}$-acetylglucosamine $(\mathrm{GlcNAc})^{[2]}$ to serine and threonine residues are key events in the cellular transduction of mitogenic signals. For instance, the serum response factor (SRF), a widely found transcription factor, is phosphorylated at various serine and threonine residues in response to extracellular stimuli. It then translocates to the nucleus, binds to the serum response element (SRE, which occurs in the promoters of numerous genes), and induces gene transcription. ${ }^{[3]}$ In addition, SRF is glycosylated at four different sites, ${ }^{[4]}$ where the major glycosylation site-most probably serine 383 in the

[*] Prof. Dr. H. Waldmann, Dr. J. Sander Institut für Organische Chemie der Universität Richard-Willstätter-Allee 2, D-76128 Karlsruhe (Germany) Fax: (+49) 721-608-4825

E-mail: waldmann@ochhades.chemie.uni-karlsruhe.de

[**] This research was supported by the Fonds der Chemischen Industrie. transactivation domain-is flanked by several phosphorylation sites (Figure 1). These findings suggest that the correct orchestration of SRF phosphorylation and glycosylation as well as the controlled removal ot these covalent protein modifications appear to be paramount to the regulation of gene transcription under the control of SRF.

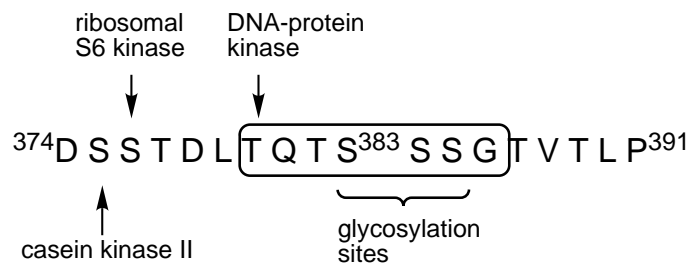

Figure 1. Phosphorylation and glycosylation sites in the transactivation domain of the serum response factor (underlined amino acid symbols). A kinase responsible for the phosphorylation of a certain amino acid is shown. The phosphorylated and glycosylated heptapeptide $\mathbf{1 7}$ synthesized in the context of this work is highlighted in the box.

Peptide conjugates embodying the characteristic structural elements of the naturally occuring protein conjugates (namely, phosphates and O-glycosides) may be efficient tools for the study of such biological processes in molecular detail. ${ }^{[5,6]}$ However, glycopeptides are very sensitive to acid and base, and phosphopeptides are even more base labile, with carbohydrate and phosphotriester groups being lost in a $\beta$ elimination reaction at $\mathrm{pH}$ values greater than $8-9 .{ }^{[5]}$ In the synthesis of glycophoshopeptides these problems potentiate each other and consequently protecting groups have to be used that can be removed with complete selectivity under the mildest conditions, preferably at $\mathrm{pH} \mathrm{6-8}$ and room temperature. ${ }^{[5]}$ We now report that such labile peptide conjugates can successfully be built up by employing the enzymatic removal of the choline ester and the $p$-phenylacetoxybenzyloxycarbonyl (PhacOZ) group as the key steps.

The choline ester was previously investigated as a possible blocking group for glycopeptide synthesis, but under the conditions required for its cleavage $(\mathrm{pH} 10-11)$ the sensitive O-glycosylated peptides were destroyed. ${ }^{[7]}$ This problem can, however, be solved efficiently by employing the enzyme butyrylcholine esterase from horse serum for the removal of the protecting group. To investigate the suitability of the enzymatic choline ester cleavage for glycopeptide synthesis O-GlcNAc-modified serine/threonine building blocks $\mathbf{1}^{[8]}$ were coupled with amino acid choline esters $2^{[7]}$ to give glycopeptides 3 in high yield. Glycodipeptide esters $\mathbf{3}$ were subsequently treated with butyrylcholine esterase in phosphate buffer at pH 6.5 (Scheme 1). In the ensuing enzymatic reaction the C-terminal choline esters were saponified exclusively. An attack on the acetate or the N-terminal urethane groups and an anomerization or $\beta$-elimination of the carbohydrate did not occur. The enzyme accepts different amino acid combinations and also tolerates sterically demanding residues such as phenylalanine at the C-terminus. The desired, selectively unmasked, glycodipeptides $\mathbf{4}$ were isolated in yields of $70-80 \%$. These results clearly indicate that the enzymatic removal of choline esters can be applied advantageously to glycopeptide chemistry. Particularly re- 